\title{
A Neural Marker of Content-Specific Active Ignoring
}

\author{
Harriet A. Allen and Glyn W. Humphreys \\ University of Birmingham
}

\author{
Paul M. Matthews \\ University of Oxford
}

\begin{abstract}
The ability to attend to relevant events and to ignore irrelevant stimuli is crucial to survival. Theories disagree on whether this ability is dependent solely on increased neural activation for relevant items or whether active ignoring can also play a role. The authors examined the active ignoring of stimuli using a preview search procedure, where irrelevant faces appeared prior to relevant house stimuli. They found increased activation in brain regions associated with spatial memory and in content-specific faceprocessing areas when participants ignored the irrelevant faces. Differences arose even on trials when only previewed faces appeared, and the magnitude of these differences predicted how well faces were ignored in search. Activation associated with active ignoring decreased when a secondary task was imposed during the preview. The data reveal a neural marker for the process of actively ignoring the content and locations of irrelevant stimuli.
\end{abstract}

Keywords: preview search, fMRI, marking, inattention
The visual world contains a vast quantity of information, making it crucial for attentional mechanisms to select stimuli that are behaviorally relevant to our goals (Neisser, 1976). This selection process may involve both the active prioritizing of relevant information and the active ignoring of irrelevant information. Evidence for a process of active ignoring comes from preview search-a variation of standard visual search procedures in which distracters are staggered across time. Typically, visual search for a conjunction target (e.g., a green $H$ amongst green $A$ s and blue $H$ s) is slow and inefficient (Neisser, 1976; Treisman \& Gelade, 1980). However, presenting half the distracters as a preview at least $500 \mathrm{~ms}$ before the others improves performance, even to the level found when just the new items appear alone (Watson \& Humphreys, 1997). That the previewed items must appear for $500 \mathrm{~ms}$ or more to be discounted from search suggests a relatively slow suppressive process (Humphreys, Jung Stalmann, \& Olivers, 2004; Watson \& Humphreys, 1997). This is supported by evidence on performance when preview search is intermingled with a luminance increment detection task. Luminance increments falling at the locations of previewed distracters are more difficult to detect compared with increments falling at the locations where new items

Editor's Note. Isabel Gauthier served as the action editor for this article.-GWH

Harriet A. Allen and Glyn W. Humphreys, Behavioural Brain Sciences, School of Psychology, University of Birmingham, Birmingham, England; Paul M. Matthews, Centre for Functional Magnetic Resonance Imaging of the Brain, Department of Clinical Neurology, University of Oxford, John Radcliffe Hospital, Oxford, England.

This work was supported by the Medical Research Council, London, England.

Correspondence concerning this article should be addressed to Harriet A. Allen, Behavioural Brain Sciences, School of Psychology, University of Birmingham, Birmingham B15 2TT, England. E-mail: H.A.Allen@bham.ac.uk will appear or at unoccupied (neutral) locations (Agter \& Donk, 2005; Humphreys et al., 2004; Olivers \& Humphreys, 2002; Watson \& Humphreys, 2000). This decrease in detection grows as the preview appears for longer, suggesting the involvement of a suppressive process that increases over time (Braithwaite, Humphreys, \& Hulleman, 2005; Humphreys et al., 2004). In addition to this, the benefit to search performance under preview conditions decreases when attention is occupied by another task during the preview period (Humphreys, Watson, \& Jolicoeur, 2002; Olivers \& Humphreys, 2002; Watson \& Humphreys, 1997); in contrast, introducing a secondary task before search has minimal effects in baseline conditions when all the items appear simultaneously. Apparently, observers have to actively engage in ignoring the old items to reap maximum benefit.

If items can be deprioritized for selection by active ignoring, one important question is what type of information is suppressed during this process. Behavioral evidence from dot probe tasks suggests that information from the locations of irrelevant items is suppressed (Humphreys et al., 2004; Olivers \& Humphreys, 2002; Watson \& Humphreys, 2000), and there is also evidence for the suppression of other properties of the stimuli; for example, detection of the target in preview search is disrupted when the target carries feature values common to the ignored preview (e.g., having the same color; Braithwaite \& Humphreys, 2003; Olivers \& Humphreys, 2003). This last result suggests that inhibition of the properties of previewed distracters can be carried over to new items with the same properties (Braithwaite et al., 2005; Olivers, Humphreys, \& Braithwaite, 2006). It is unclear, however, whether there is an additional suppression based on the contents of the ignored stimuli-for example, of particular objects or classes of objects and not just their feature values. This is investigated here. We use functional brain imaging to probe the activation in brain regions responding specifically to the content of the preview, and we assess whether there are activation changes linked to when the preview is being actively ignored. This examination is directly relevant to theories of how the preview effect comes about. Although we have noted evidence for the active suppression of the 
preview, other authors have argued that the preview benefit in search is driven solely by attention to the new stimuli. In particular, attention may either be captured by the new onsets of the search stimuli (Donk \& Theeuwes, 2001) or there may be automatic temporal segmentation of the preview and search displays and then selective allocation of attention to the search items (Jiang, Chun, \& Marks, 2002). If this is the case, however, then we would not expect activation changes in brain regions responding to the content of the preview specifically under conditions in which the preview is being actively ignored. In contrast, such changes may provide a marker of active ignoring of the preview.

\section{Visual Working Memory Interferes With the Preview Benefit}

As noted above, evidence for a role of active processes in creating the preview benefit in search comes from experiments on secondary task interference effects, where the benefit is disrupted when a secondary task is performed during the preview period. At least some aspects of this interference effect are modality specific. Humphreys et al. (2002) varied the modality of the secondary task. In one case, participants had to shadow a stream of digits presented at fixation while the preview was displayed. In a second condition, an auditory shadowing task was carried out across the same time period. Furthermore, the shadowing task either started at the same time as the preview was displayed or began only after the preview had been displayed for a period. Humphreys et al. found equivalent effects of the visual and auditory secondary tasks when these tasks started at the onset of the preview. However, only the visual secondary task disrupted search when this task began after the preview had already been exposed (the auditory secondary task then had minimal effect). To account for these results, Humphreys et al. proposed that secondary tasks could have (at least) two effects on performance. Secondary tasks that begin concurrently with the preview affect how well the preview is encoded, and this occurs irrespective of the modality of the secondary task. Once the preview has been encoded, though, only the visual secondary task disrupts performance because it affects how well the representation of the preview is maintained. According to this account, encoding and maintenance of a representation of the preview is necessary to enable the preview to be filtered out from search. Translated into neural terms, the selective effect of the visual secondary task may be attributable to items in the secondary task and items in the preview competing for the same neural space, disrupting the preview's representation. This was evaluated here. We introduced a condition in which participants carried out a visual memory (VM) task along with having to carry out a preview search task. We assessed whether the VM task disrupted the preview benefit in search and whether the memory task was associated with activation in brain regions also associated with responding to the preview.

Instead of arguing that the secondary task disrupts the active ignoring of the preview, it alternatively can be argued that the secondary task makes it more difficult for participants to generate an expectancy for the search display on the basis of the preview and that loss of this expectancy is responsible for secondary task interference (see also Braithwaite \& Humphreys, 2003; Humphreys et al., 2004, for evidence of a positive role of expectancy in preview search). However, if this were the case, then we would not necessarily expect activation associated with the memory task to be found in the same neural regions linked to the preview. Moreover, activity in content-specific regions of the brain should be associated with the expectancy for the search items and not the contents of the preview.

\section{The Present Study}

We report behavioral and functional magnetic resonance imaging (fMRI) data from a preview search task. The search task was performed simultaneously with the fMRI data acquisition. The behavioral task used face and house stimuli. Participants searched for a blue house presented among red house distracters. In the preview condition, these search stimuli were preceded by blue faces in a preview display, which remained present when the search display was presented (see Figure 1A). In the full-set baseline condition, participants searched for a blue house among red houses and blue faces, and all these items appeared simultaneously. In the half-set baseline condition, participants searched for a blue house among red houses. In both the full- and half-set conditions, the search displays were preceded by an initial display of faces, similar to that used for the preview. However, these initial faces disappeared on presentation of the search displays and thus conveyed no information relevant for the subsequent search task. The full-set and half-set baseline conditions were interleaved in the same block (see Figure 1B). The preview search condition was performed in a separate block. Crucially, participants knew which condition they were in, thus they knew whether the first display was a preview or a noninformative dummy preview. In the full-set baseline, the number of items presented in the to-be-searched display matched the number of items presented in the final display in preview search. Any benefit from the preview conditions should lead to an improvement in the preview condition compared with the full-set baseline. In the half-set baseline, the number of items in the search display matched the number of new items appearing in the preview condition. If there is no interference from these previewed distracters on search performance, then performance in the preview condition should match this baseline. The results of this experiment allow us to generalize the preview benefit to displays that are more complex than those typically used in prior studies.

To investigate brain activation in preview search in more detail, in the preview full-set and half-set search conditions we interspersed the trials described above with occasional unexpected trials in which only the preview (or dummy) was presented and no search display appeared (the preview-only or dummy-only conditions). In these trials, the visual stimulus was the same between the conditions and only the expectation or attentional set was different. We used a region of interest analysis to assess the relative blood oxygen level dependent (BOLD) fMRI activation in contentspecific face-processing areas during both preview search and trials where only the preview display appeared. This activity was compared with activation in full-set (blue house target vs. blue faces and red houses presented simultaneously) and half-set (blue house target vs. red house distracters) baselines, both when the full series of displays was shown and when just the first dummy display was shown. Unlike in the preview condition, the faces in the dummy display were unlikely to be actively ignored in the fulland half-set baselines. If the initial faces are actively ignored in the 


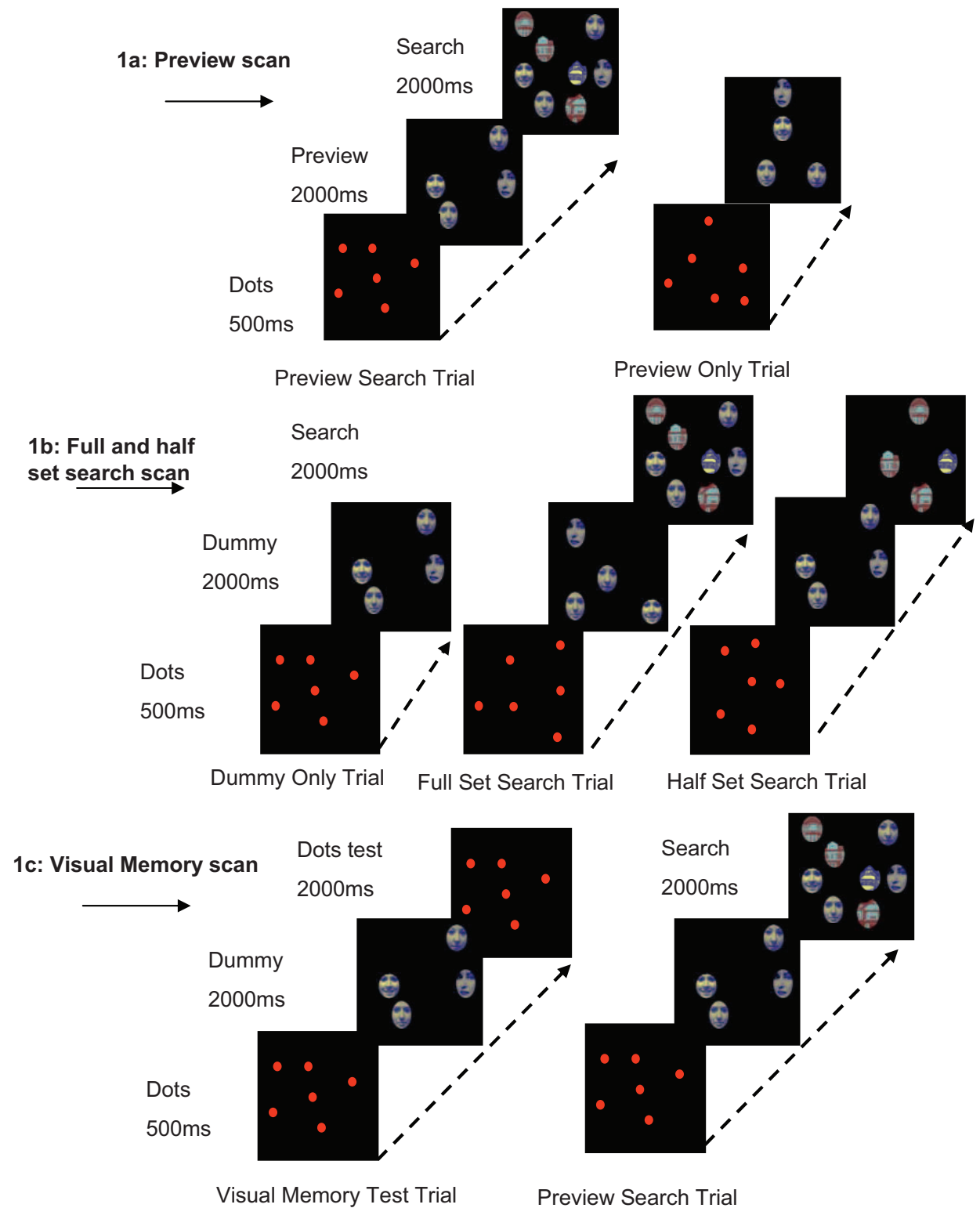

Figure 1. Illustration of trial conditions in the three search scans. A: Preview scan. Participants were told to find the blue house in the third display and that the target would never appear among the faces, that the faces would remain on the screen throughout the trial, and that the dots were irrelevant. B: Half- and full-set search scan. Instructions were the same as those for the preview scan except that participants were told that the faces in the second display were not relevant to the task. C: Visual memory scan. Participants were told to memorize the initial dot pattern and to indicate whether the second dot display was the same as or different from the first display. When a search display was presented, participants switched tasks to find the blue house.

preview but not the other conditions, then neural activity should differ in preview compared with full- and half-set search, and this should hold especially for comparisons between preview-only and dummy-only trials when exactly the same displays are presented in the preview and search conditions.

In addition to these search conditions, we also examined behavior and neural activity when participants engaged in a VM task while the preview displays appeared. In the VM condition, participants had to remember the locations of a set of dots that appeared prior to the initial face display. On half of the trials, the faces were replaced by another set of dots and participants had to decide whether one of the dots had changed its position. On the other half of the trials, the faces remained in the display and a blue house target was presented along with red house distracters (see Figure 1C). When this occurred, participants were instructed to search for the blue house target. So that all conditions were equal, an initial set of dots appeared on all trials in all conditions, though these items were completely irrelevant in the nonmemory blocks. Prior 
behavioral evidence suggests that preview search can be selectively disrupted when participants engage in a secondary task during the preview display, consistent with a process of active ignoring being impeded (Humphreys et al., 2002). It follows that any activation change associated with active ignoring should be reduced on search trials in the VM block. Activity associated with VM itself was measured against activity when participants fixated a central cross. This allowed us to establish whether the regions activated in preview search overlapped with those usually associated with VM.

Prior studies of preview search using brain imaging have shown increased activation of the superior parietal lobule (SPL) and precuneus in the preview relative to full- and half-set baselines (Olivers, Smith, Matthews, \& Humphreys, 2005; Pollmann et al., 2003). Though this suggests that these brain regions mediate the active ignoring of old items, there is no converging evidence that reductions in this activation are linked to decreases in the benefit. We assess this here by examining activation under dual- as well as single-task conditions. Of more importance, we evaluated whether active ignoring spread to the content of any representations by examining activation in cortical regions responsive to the particular stimuli presented (e.g., in the fusiform gyrus, which is sensitive to faces; Kanwisher, McDermott, \& Chun, 1997). We ask whether active ignoring is modulated by a network of regions, including those concerned with representing the spatial layout of the ignored items (e.g., SPL and precuneus) and those concerned with representing the content (e.g., the fusiform gyrus). Converging evidence for spatial representation in the parietal regions was sought by examining activations directly in our VM-loaded preview condition.

BOLD activation has been shown to increase in content-specific neural regions when attention is directed to stimuli that activate these areas. For example, in a display containing overlapping face and house images, activation increased in face or house processing areas when attention was directed to that aspect of the image (O'Craven, Downing, \& Kanwisher, 1999). When stimuli are unattended, there can also be decreased activation in the contentspecific region. Gazzaley, Cooney, McEvoy, Knight, and D'Esposito (2005) showed participants a sequence of landscapes and faces with instructions to attend to one category or the other. When attending to faces and ignoring landscapes, activation in face-processing areas increased and activation in place-processing areas decreased compared with a baseline in which both stimuli were attended. However, any decrease in activation could reflect increased processing in the attend condition rather than active suppression of distracters. We eliminated this problem by comparing content-specific activity when previews may be actively ignored (in preview search) relative to conditions with exactly the same displays but in which the initial displays are irrelevant for search. Previous work has also compared activation when participants expect many, relative to few, distracters to be presented in a condition in which the target locations were known and attended (Serences, Yantis, Culberson, \& Awh, 2004). There was greater activation in the visual cortex on trials when many distracters were expected, suggesting that it was associated with distracter suppression and not target enhancement. However, signal enhancement and suppression were difficult to separate because the changes in activity were found in neurons responding to the locations of targets. Here, we measure activity specifically locked to the con- tent of previewed distracters, providing a clearer analysis of distracter suppression.

The behavioral evidence from preview search suggests that there might be a distinct process of actively ignoring some display items that is distinct from those items simply being viewed passively. Rather than a decrease in activation to the stimulus in this case, as might be expected when attention is removed, there might be an increase in activation to the stimulus, reflecting the fact that it is being actively inhibited.

\section{Method}

\section{Participants}

Sixteen participants $(7 \mathrm{men})$ volunteered for the study. Their ages ranged from 18 to 49 years $(M=24$ years). Three were left handed. All had normal or corrected-to-normal visual acuity. Participants received payment or course credit for taking part in the experiment. All had the procedures and risks associated with fMRI explained to them and gave informed consent to take part in the study, following the ethical procedures of the Birmingham University Imaging Centre, Birmingham, England.

\section{Stimuli}

The stimuli were images of faces and houses. All faces used in this study were monochrome images of women. These were spatially clipped to remove the hair. The original images were grayscale. For the search task, these were filtered (using MATLAB; http://www.mathworks.com) to produce output on the red or blue monitor gun only. The average luminance of the sets of blue and red faces was matched over the group. Houses were monochrome photographs of houses in a style familiar to the participants (typical Birmingham, England houses). For the search task, the images were clipped to match the shape and average luminance of the set of face images and filtered to be either red or blue in the same way as the face images. There were 40 face images and 40 house images. Whenever an image of a particular type was presented, it was selected at random, with replacement, from the appropriate set (see Figure 1 for examples of the stimuli).

\section{Procedure}

There were four functional scans. In three of these, the participants performed a search task. Their task was to indicate, with a button press, whether the blue house target was to the left or right of a fixation cross. Diagrams of the trial procedure are shown in Figure 1, and the details of the individual scans are explained below. All scans began with a 10-s fixation and then a 4 -s presentation of reminder instructions. Subsequently, trial presentations were synchronized to the scanner pulse, which occurred on every volume acquisition (time to repetition [TR] $=2 \mathrm{~s}$, except where noted). Further details of scanning are below. All four scan tasks were practiced, in full, in the week prior to the scan and were reexplained to the participant during the scanning session. All participants performed at $80 \%$ correct or better in the practice session (once they had learned the task).

Preview search scan. There were two types of trials in this scan block (see Figure 1A). The majority (40) of trials comprised preview search. Participants viewed a display of six dots $(500 \mathrm{~ms})$, 
presented to maintain consistency between the visual displays in all the scans, followed by a display of four or eight blue faces ( 2 s); these faces remained on the screen and were joined by three or seven red houses and one blue house ( $2 \mathrm{~s})$. During the presentation of the second display (faces and houses together), participants indicated (with a keypress) whether the blue house was to the right or left of fixation. Feedback was given during the intertrial interval. If participants took longer than $2 \mathrm{~s}$ to respond, then this was counted as an incorrect trial. Participants were told to ignore the faces, that these would remain on the screen and never contain a target, and that the target item was always in the second group on each trial. Participants were asked to keep accuracy as high as possible. On a minority of (20) trials, participants were presented with the preview display only. In this condition, the dot display was followed by the faces but there was no search display. Participants were told not to respond on the occasions when this happened. The order of the trials was randomly ordered, separately for each participant; thus, participants should have been in the same state during the preview-only and full preview trials. To improve the power of the functional imaging data, we set the intertrial interval to between 4 and $12 \mathrm{~s}$, randomly ordered.

Full- and half-set search task scan. There were three types of trials in this scan block (see Figure 1B). All trials began with the presentation of six red dots (not relevant to the task in this scan), followed by a display of four or eight blue faces. On all trials, these faces then disappeared. On the majority of trials (58), a search display containing new distracter items in new positions was presented immediately afterward, and the task was to locate the blue house. There were two search conditions. In the half-set baseline, the distracters were red houses only (four or eight items in total). This condition matched the preview search condition in terms of the number of new search items presented. In the full-set baseline, the distracters were red houses as well as blue faces ( 8 or 16 items in total). On a minority of trials (29), no search display was presented. Other details were as above.

VM and loaded preview scan. There were two types of trials in this block (see Figure 1C). On half of the trials (29), participants performed a VM task. They viewed a display of six dots $(500 \mathrm{~ms})$, followed by a display of four or eight blue faces ( $2 \mathrm{~s})$ and then by a second display of dots $(2 \mathrm{~s})$. This second dot display could be identical to the first display (same trials) or one dot could be repositioned on the screen (different trials). Participants made a button press to indicate whether the first and second dot displays were the same or different. Participants were told to prioritize this VM task and to aim to achieve $100 \%$ correct. On the remainder of the trials, participants undertook a preview search task (the loaded preview condition). On these trials, the first dot display was followed by the faces, and the faces were then joined by red houses and a blue target house. Whenever a search display appeared instead of a second display of dots, participants were instructed to search for a blue target house. Other details were as above.

Localizer for face and house areas. Participants viewed faces, houses, or a fixation cross presented at fixation. Images were grayscale versions of the images used in the experiment. They were twice the size of those used in the experiment. House images were not spatially clipped. This scan followed a blocked design. For the first $9 \mathrm{~s}$ of the cycle (synchronized to the scanner pulse), participants viewed a fixation cross that changed between red and blue; this was followed by $18 \mathrm{~s}$ of pictures of faces that changed identity every $3 \mathrm{~s}$, then a further $9 \mathrm{~s}$ of the color changing fixation cross followed by $18 \mathrm{~s}$ of pictures of houses that changed identity every $3 \mathrm{~s}$. This cycle was repeated 10 times. Throughout the scan, participants performed a one-back matching task on either the fixation cross or the identity of the face or house. The identity of the face and house, and the color of the fixation cross, remained the same on one third of trials.

Imaging methods. The imaging data were collected on a GE 3T MRI scanner (Chalfont St. Giles, Bucks, England) using a head coil. Each participant lay in the scanner wearing prism glasses to view a screen at their feet onto which the images were back projected. During the session, there were four functional scans (see above) acquired using a quiet echo planar sequence (EPI). Three of these followed an event-related design. Each volume consisted of $253-\mathrm{mm}$ slices in the coronal plane covering the posterior half of the brain. Technical limitations meant that we were unable to image the full brain at the required resolution; ongoing work in our laboratory is investigating more anterior activation found with this task. For the preview search, full- and half-set search and VM scan volumes were acquired with a TR of $2 \mathrm{~s}$, time to echo (TE) $=30$ ms, spin angle $=79^{\circ}$, field of view $=256 \times 192$, matrix $=64 \times$ 64. For the localizer scan, the TR was $3 \mathrm{~s}$ but all other parameters were the same. Anatomical scans were acquired during the same session for all but 4 participants (their structural scans had been acquired in a previous session). Structural scans were acquired using turbo flash sequences, with TR $=20 \mathrm{~ms}$ and 30 slices of 2 $\mathrm{mm}$ thickness in the coronal direction $(\mathrm{TE}=5 \mathrm{~ms}$, time to inversion $=500 \mathrm{~ms}$, flip angle $=12^{\circ}$, field of view $=128 \times 128$ $\mathrm{mm})$.

Data analysis. The fMRI data were analyzed using the fMRI Expert Analysis Tool (FEAT) Version 5.4, part of FMRIB's Software Library (available from www.fmrib.ox.ac.uk/fsl). The first six volumes of the search task scans (12 s) were removed to reduce the transient effects of magnetic saturation and to allow the hemodynamic response to stabilize. The first five volumes were removed from the localizer scans (15 s). Individual scans were preprocessed in the following ways before further analysis. Slicetiming correction (using Fourier-space time series phase shifting) was applied. Movements of the participants' heads were corrected for using MCFLIRT (Jenkinson, Bannister, \& Smith, 2005). Average head movement by participants was low $(0.33 \mathrm{~mm}$; range $=$ $0.09-0.89 \mathrm{~mm})$. The skull and other nonbrain matter was removed using BET (S. Smith, 2002). Images were then spatially smoothed using a Gaussian kernel of full width at half maximum (FWHM) $7 \mathrm{~mm}$, and all volumes within a scan were mean-based intensity normalized. Signals within in the scan were then high-pass temporal filtered (Gaussian-weighted LSF straight line fitting, with sigma $=37.5 \mathrm{~s}$ ). Each participants' partial head EPI image was registered to a whole head EPI image (acquired immediately after the functional scans in the same session). This was then registered to their individual (brain extracted) structural images. The individual scans were then registered to high-resolution standard images in Montreal Neurological Institute (MNI) space using FLIRT (Jenkinson et al., 2005; Jenkinson \& Smith, 2001).

Time-series statistical analysis on individual data (see below for exact comparisons) was carried out using FILM with local autocorrelation correction (Woolrich, Ripley, Brady, \& Smith, 2001). The study had an event-related design, with an event being defined as the full duration of a trial (i.e., dot display + preview/dummy + 
search). Higher level analysis was carried out using FMRIB's Local Analysis of Mixed Effects (FLAME; Beckman, Jenkinson, \& Smith, 2003; Woolrich, Behrens, Bedell, Jenkinson, \& Smith, 2004). $Z$ (Gaussianized T/F) statistic images were thresholded using clusters determined by $Z>2.3$ and a (corrected) cluster significance threshold of $p=.05$, unless otherwise stated (Worsley, Evans, Marrett, \& Nelson, 1992). To identify regions significantly activated by faces or houses in the localizer scan, we thresholded individual data statistical images at $p=.01$ (uncorrected). Face-specific responses in the fusiform gyrus were found in both hemispheres of 9 participants, only in the left hemisphere of 1 participant, and only in the right hemisphere of 2 participants. Registration to high-resolution and/or standard images was carried out using FLIRT. Time courses were extracted for each participant separately and then averaged using PEATE (supplied by J. Kaplan, http://www.jonaskaplan.com/peate.html).

\section{Results}

\section{Behavioral Data}

Of the original 16 participants, 3 were excluded from further analysis because they did not show a clear preview benefit. ${ }^{1}$ The remaining 13 participants all achieved greater than $75 \%$ correct while performing the search task in the scanner (mean proportion correct for each condition is shown in Table 1).

\section{Memory Task}

One further participant was excluded from analysis for the VM scan only, as he or she did not perform above criterion $(75 \%$ correct) on that task in the scanner. The mean percentage of correct responses on the VM task was $88 \%$ (range $=75 \%-97 \%$ ).

\section{Errors}

The proportion of correct responses in each search condition is shown in Table 1 . A $3 \times 2$ within-subjects analysis of variance (ANOVA) comparing condition (preview, full-set, and half-set baselines) and number of distracters found a significant effect of condition, $F(2,24)=5.9, p=.009$, partial $\eta^{2}=0.33$. The effect of the number of distracters narrowly missed significance, $F(1$, $12)=4.3, p=.06$, partial $\eta^{2}=0.26$. The interaction was not significant, $F(2,24)=1.6, p=.3$, partial $\eta^{2}=0.12$. This pattern of results is unsurprising, given that the participants had a restricted duration in which to respond and reaction times (RTs) are typically longer when there are more items presented and when conjunction search is carried out (as in the full-set condition). A second $3 \times 2$ ANOVA comparing the proportion of correct responses in the loaded preview condition and the full- and half-set

Table 1

Proportion of Correct Responses in Each Condition of the Experiment in the Scanner

\begin{tabular}{lcccc}
\hline $\begin{array}{c}\text { No. of } \\
\text { items }\end{array}$ & Preview & $\begin{array}{c}\text { Loaded } \\
\text { preview }\end{array}$ & Full set & Half set \\
\hline 8 & .98 & .99 & .93 & .97 \\
16 & .96 & .89 & .89 & .97 \\
\hline
\end{tabular}

baselines found a significant effect of the number of distracters, $F(1,11)=8.8, p=.013$, partial $\eta^{2}=0.45$, as well as an effect of condition, $F(2,22)=5.3, p=.013$, partial $\eta^{2}=.32$, and a significant interaction, $F(2,22)=6.5, p=.006$, partial $\eta^{2}=0.37$. Again, this is unsurprising, given the limited duration available for responses. Accuracy dropped particularly in the loaded preview and full-set conditions.

\section{$R T s$}

We recorded accuracy and RTs while scanning for all participants (although technical failure meant that RT data were lost for the loaded preview condition for 1 participant). Accuracy was high (see Table 1) but fell when the RTs increased. This is likely due to the response deadline necessary in the scanner, which recorded all responses over 2,000 $\mathrm{ms}$ as incorrect. Therefore, we used response efficiency as the dependant measure in our analysis. For each participant separately for each condition, the RT was divided by accuracy in that condition. This adjustment was originally proposed by Townsend and Ashby (1983) and has recently been used by Mevorach, Humphreys, and Shalev (2006) and Chambers, Mark, Stokes, Janko, and Mattingley (2006) in experiments in which high error rates were induced. Figure 2 shows the efficiency measure for each condition, plotted against the number of items. The number of items displayed in the searched display of the half-set condition was half that in the full-set and preview conditions; however, the half-set condition matched the preview condition in terms of the number of new items presented in the second display. To allow visual comparison between the conditions, we presented the data in two subplots. Figure 2A plots efficiency in the full set, preview, and loaded preview against the total number of items visible in the second searched display. Figure 2B plots efficiency in the half set, preview, and loaded preview against the number of new items presented in the second display.

To establish whether the preview display benefited performance, we compared the data from the preview display with that from the full- and half-set conditions. There was a significant effect of condition (preview, full set, half set), $F(2,24)=21.3$, $p<.0005$, partial $\eta^{2}=0.64$, and of the number of search items, $F(1,12)=52.9, p<.0005$, partial $\eta^{2}=0.82$. There was also a significant interaction, $F(2,24)=5.2, p=.013$, partial $\eta^{2}=0.3$. To investigate these effects and interactions further, we conducted additional two-way ANOVAs. There was a significant difference between the full-set and preview conditions, $F(1,12)=27.9, p<$ .0005 , partial $\eta^{2}=0.7$, and a significant interaction between these two conditions, $F(1,12)=4.8, p=.048$, partial $\eta^{2}=0.29$. Comparing preview with half set, there was no significant main effect of condition, $F(1,12)=0.9, p=.36$, partial $\eta^{2}=0.071$, and no significant interaction, $F(1,12)=3.7, p=.078$, partial $\eta^{2}=0.23$. Thus, performance in the preview condition did not differ significantly from the half-set condition, but search effi-

\footnotetext{
${ }^{1}$ Similar to many behavioral studies, not all participants demonstrate a reliable effect of the conditions, though the effect is highly reliable across participants. However, because we were interested in establishing the neural basis of the preview benefit, the small minority of participants who failed to show a behavioral effect in the scanner were not relevant to the fMRI analysis. We note, however, that the behavioral results remained reliable even when these 3 participants were included.
} 
a)
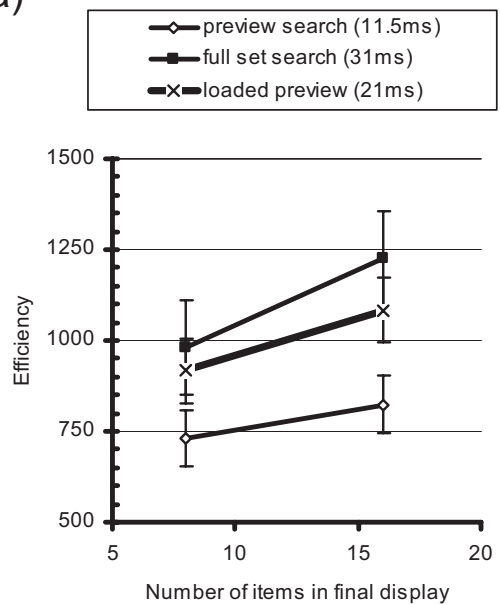

b)
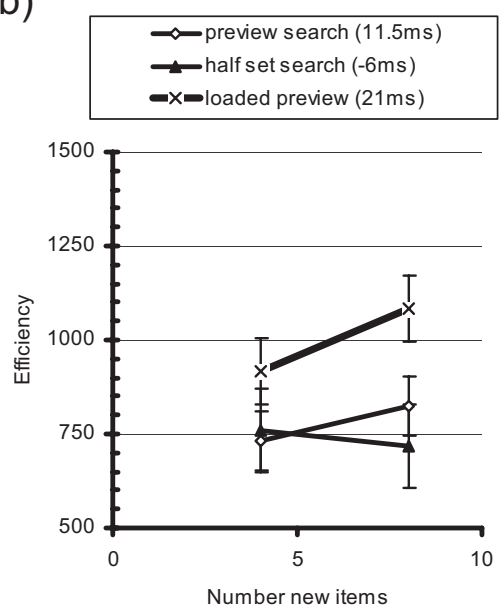

Figure 2. Efficiency (in milliseconds) for the search task in the scanner plotted against number of items. Panel A shows efficiency as a function of the total number of items in the preview or full-set conditions, and Panel $\mathrm{B}$ shows efficiency as a function of the number of new items in the preview and half-set conditions (efficiency $=$ reaction time/error). Participants searched for a blue house among red houses (half set; triangles), a blue house among red houses and blue faces (full set; squares), a blue house among red houses and blue faces where the faces were previewed (preview; diamonds), or a blue house in the preview display while also performing a visual memory task on a dot pattern (loaded preview; crosses). Error bars are \pm 1 standard deviation of the mean. Times per search item are shown in the legend.

ciency was better in the preview than in the full-set condition. This is the classic preview benefit.

We expected that the preview benefit would be reduced under conditions when the preview is presented with a VM load. We first compared performance in the loaded preview condition with the full- and half-set conditions. There was a significant effect of condition (loaded preview, full set, half set), $F(2,22)=16.2, p<$ .0005 , partial $\eta^{2}=0.6$, and number of items, $F(1,11)=293.0$, $p<.0005$, partial $\eta^{2}=0.73$, as well as a significant interaction, $F(2,22)=4.5, p=.025$, partial $\eta^{2}=0.289$. To investigate the interaction further, we conducted additional two-way ANOVAs. There was no significant difference between the loaded preview and full-set condition, $F(1,11)=3.9, p=.075$, partial $\eta^{2}=0.26$, and no significant interaction with display size, $F(1,11)=1.7$, $p=.22$, partial $\eta^{2}=0.13$. There was, however, a significant difference between the loaded preview and half-set condition, $F(1$, 11) $=17.5, p<.002$, partial $\eta^{2}=0.61$, and the Condition $\times$ Display Size interaction approached significance, $F(1,11)=4.3$, $p=.061$, partial $\eta^{2}=0.28$. Performance with the loaded preview tended toward that in the full-set condition rather than the half-set baseline. Finally, we compared the loaded preview and standard preview conditions. There was an overall difference between these two conditions, $F(1,11)=50.7, p<.0005$, partial $\eta^{2}=0.82$, but no significant interaction with display size, $F(1,11)=2.3, p=$ .16, partial $\eta^{2}=0.17$, though the pattern of results is in the direction we predicted (the display size effects in the loaded condition were almost twice those in the standard preview condition).

\section{Imaging Data}

Our experiment contained three complementary contrasts designed to isolate activations relating to the preview benefit on search. First, we contrasted the preview-only trials (during the preview block) with the dummy-only trials (during the full- and half-set search block; i.e., preview only - dummy only). These two conditions differed only in the participants' knowledge of the display; the visual content of the displays (face images) and the task (no response) were identical. Any difference in activation, therefore, must be due to the processes underlying the preview benefit. Results from this comparison offer the strongest indication of the brain's behavior during the preview phase of a search trial. In two further contrasts, we assessed activation in preview search relative to each of the full- and half-set baselines (i.e., preview full-set search, preview - half-set search). We also contrasted the two conditions in which participants successfully performed preview search with all the conditions in which they did not (i.e., [preview only + preview search] - [half-set search + full-set search + dummy only]). Activations relating to the preview benefit should be found in (a) the preview only - dummy only contrast; (b) the conjunction of preview only - dummy only, preview - full set, and preview - half set; and (c) the contrast of (preview only/preview search) - (dummy only/half set/full set).

Parietal and occipital activations related to the preview benefit. The contrasts described above were performed across the full acquisition volume (see Table 2 and Figures 3A, 3B, and 3C). A conjunction analysis (as described above) revealed four regions that were active in all three contrasts (see Table 2). These were in the left precuneus, the right precuneus extending into the cuneus, the left cuneus, and the right cerebellum. Finally, the contrast of all preview to all nonpreview conditions revealed activations in the left and right inferior parietal lobes (see Table 2 and Figure 3D, all preview - all nonpreview).

Response to preview in stimulus-specific areas. The main focus of this study was on the change in stimulus-specific activation 
Table 2

Coordinates of Significant Activations

\begin{tabular}{|c|c|c|c|}
\hline Condition and Structure & Location $(x, y, z$ of maxima) & $Z \max$ & Vol (vox) \\
\hline \multicolumn{4}{|l|}{ Preview only - dummy only } \\
\hline Left cerebellum VI** & $-30,-70,-30$ & 4.07 & 2,815 \\
\hline $\begin{array}{l}\text { Left precuneus (BA7), extending into right and } \\
\text { left cuneus (BA18) }\end{array}$ & $\begin{array}{c}-6,-82,40 \text { to } 10,-78,44 \text { and }-4, \\
-88,16\end{array}$ & $4.81,4.23,4.33$ & 1,386 \\
\hline \multicolumn{4}{|l|}{ Preview-full-set search } \\
\hline Right cerebellum IV & $20,-42,8$ & 3.97 & 2,597 \\
\hline Left cuneus, ${ }^{*}$ right cuneus ${ }^{*}$ & $0,90,20 ;-4,-88,22 ; 8,-88,34$ & $4.77,4.71,4.12$ & 779 \\
\hline \multicolumn{4}{|l|}{ Preview-half-set search } \\
\hline Left precuneus ${ }^{* *}$ extending to left cuneus & $-6,-80,40$ to $-20,-88,32$ & $6.37,5.23$ & 15,492 \\
\hline \multicolumn{4}{|l|}{ All preview-all nonpreview } \\
\hline Right inferior parietal lobe in precuneus ${ }^{* *}$ & $40,-68,44$ & 5.56 & 1,465 \\
\hline Left inferior parietal lobe ${ }^{* *}$ & $-44,-66,30$ & 5.79 & 1,388 \\
\hline Right, left cuneus and precuneus $(B A 7,19)^{*}$ & $0,-88,14$ & 5.91 & 993 \\
\hline Cerebellum V* & $-4,-86,-21$ & 5.89 & 914 \\
\hline \multicolumn{4}{|l|}{ Visual memory } \\
\hline Brainstem $* *$ & $-6,-30,-20$ & 6.5 & 1,612 \\
\hline Left inferior temporal gyrus (BA18) ${ }^{* * *}$ & $-50,-52,-18$ & 7.04 & 1,305 \\
\hline Right cerebellum ${ }^{* *}$ & $10,-80,-20$ & 9.2 & 953 \\
\hline Left precuneus (BA19) ${ }^{* *}$ & $-28,-82,-24$ & 9.92 & 933 \\
\hline \multicolumn{4}{|l|}{ Loaded preview search } \\
\hline Right lingual gyrus ${ }^{* * *}$ & $14,-88,-18$ & 10.9 & 10,667 \\
\hline Left precuneus (BA19) ${ }^{* * *}$ & $-24,-84,40$ & 9.56 & 9.56 \\
\hline
\end{tabular}

Note. Location coordinates are in millimeters. IV, V, and VI cerebellum areas are as defined in Schmahmann, Doyon, Toga, Petrides, and Evans (2000). $\mathrm{BA}=$ Brodmann area; $Z \max =\operatorname{maximum} Z$ score; $\mathrm{Vol}=$ volume; vox $=$ voxel. ${ }^{*} p<.05 .{ }^{* * *} p<.01$.

related to a preview benefit in search. Participants underwent a localizer scan for areas activated by our face stimuli. We extracted time courses of activation from these areas for each condition (see Figures 4A-4C). In all cases, the activation levels for the preview condition were higher and earlier than the activations for the nonpreview condition. ANOVAs were performed over the first $16 \mathrm{~s}$ of the time course. Activation in the preview-only conditions was greater than in the dummy-only condition in both hemispheres (see Figure 4A): right, $F(1,10)=5.1, p=.048$, and left, $F(1,9)=$ $5.8, p=.039$. For the trials in which search occurred, the difference in activation between the preview and the half-set baseline was also significant in both hemispheres (see Figure 4C): right, $F(1,10)=5.6, p=.04$, and left, $F(1,9)=12.0, p=.007$. The same pattern of difference was apparent between the preview search condition and full-set search, but here it was not significant. It should be noted, however, that in the full-set condition, additional face onsets added to the activation, possibly overshadowing any increase due to the preview. Nevertheless, the contrast between the preview-only and dummy-only conditions, in particular, suggests earlier and enhanced activation to the to-be-ignored set of faces under conditions of preview search.

VM scan. Activity linked to VM (i.e., the VM task vs. the fixation baseline; see Table 2 and Figure 3E) was found in the precuneus and inferior temporal gyrus, close to areas active in previous studies (Pessoa, Gutierrez, Bandettini, \& Ungerleider, 2002). On some trials in the same block, participants had to perform a preview search task instead of the VM task. On these trials, the stimuli were identical to those used in the preview search trials of the preview scan. Activations for this loaded preview task (loaded preview - fixation) were found (see Table 2) and appeared to overlap with those found for standard preview search. This was confirmed by a conjunction analysis involving the loaded preview and standard preview search conditions. This showed that regions centered on the right precuneus $(1,-74,44)$, left cuneus $(-3,-82$, $23)$, right cuneus $(2,-88,8)$, and right culmen $(10,-64,-16)$ were significantly active in both contrasts; however, only in the preview scan was a behavioral preview effect observed.

Figure 4D shows the average time series extracted from facespecific regions of interest in the loaded preview condition compared with that found under standard preview conditions. Activation in the standard preview condition was higher than in the loaded preview condition in the right hemisphere, despite an extra VM load being carried under the latter condition: right, $F(1,6)=$ $12.0, p=.039$, and left, $F(1,4)=2.0, p=.228$. Activation was not significantly changed in the left hemisphere under load conditions; however, there were only 5 participants who fulfilled all the criteria for this comparison.

Correlations of brain activity with behavior. The increased activation in the face-specific region of interest when the preview was used successfully may reflect processes involved in actively ignoring the preview display. If this is the case, then we would predict a correlation between the magnitude of the preview benefit and the increase in activation. This difference is likely to be found only when comparing preview-only and dummy-only scans because only in these two conditions was the stimulus perfectly matched. We quantified the magnitude of the behavioral preview benefit separately for each participant using the following equation:

$$
P V=\frac{\text { (efficiency: full set })-(\text { efficiency: preview })}{(\text { efficiency: preview })} .
$$

These values are plotted in Figure 5 for each participant separately against the average difference between the two times-series curves. 
3a: Preview only - dummy only

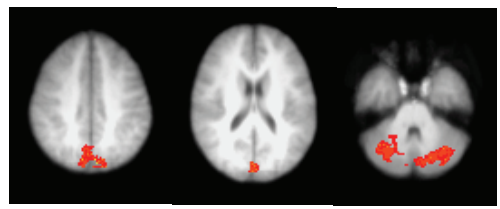

3b: Preview search - full set search

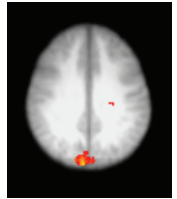

3c: Preview search - half set search

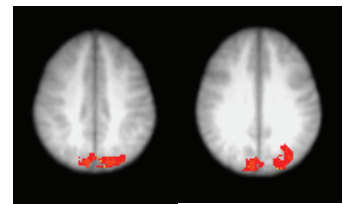

3d: All preview - all non-preview

3e: Visual memory task

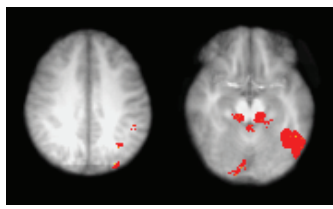

Figure 3. Brain regions showing significant activations from the group analysis overlaid on the Montreal Neurological Institute (MNI) template brain. It is important to note, however, that our functional scans did not include the anterior half of the brain. A, B, and C: Activations from the three whole brain contrasts designed to find preview-related activation (i.e., preview only - dummy only, preview search - full-set search, preview search - half-set search). D: Areas showing activation in the subtraction of all nonpreview conditions from all preview conditions. E: Areas activated by the visual memory task (vs. fixation). We did not scan sufficiently anteriorly to find the activations in the dorsolateral prefrontal cortex or frontal eye fields that are commonly associated with this type of task.

The behavioral preview benefit was significantly correlated with activation in the fusiform face area in the left hemisphere (Pearson's $\left.R^{2}=.626, p=.027\right)$ but not in the right hemisphere $\left(R^{2}=\right.$ $.111, p=.373)$. It is surprising that no significant correlation was found in the right hemisphere because a significant overall activation effect was found in both hemispheres. Caution must be used in interpreting the results of correlations of small data sets such as these; however, the finding is corroborative of our main finding, and stronger activation in the left than in the right hemisphere parietal areas during preview search has been previously reported (Pollmann et al., 2003)

\section{Discussion}

The behavioral data replicated the standard preview benefit on search performance. Participants were able to exclude the previewed items. In addition, preview search efficiency was disrupted in the memory load condition, when items were held in visual short-term memory prior to the preview appearing. This last result is noteworthy. In prior studies of visual secondary task interference on preview search, the secondary task typically required participants to focus their attention at the center of the display to shadow a stream of briefly presented digits. This may demand that participants adopt a narrow tuning of attention, reducing attention to the preview stimuli falling at more peripheral locations (Lavie, 1995; Yantis \& Jonides, 1990). Here, however, participants had to spread their attention across the display to encode and maintain the random array of dots. Despite being encouraged to adopt a distributed mode of attention, the secondary task interfered with search. Hence, the effects of the secondary task do not seem
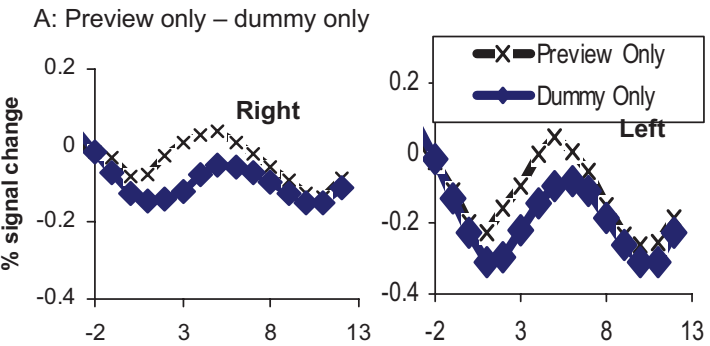

B: Full set - preview search
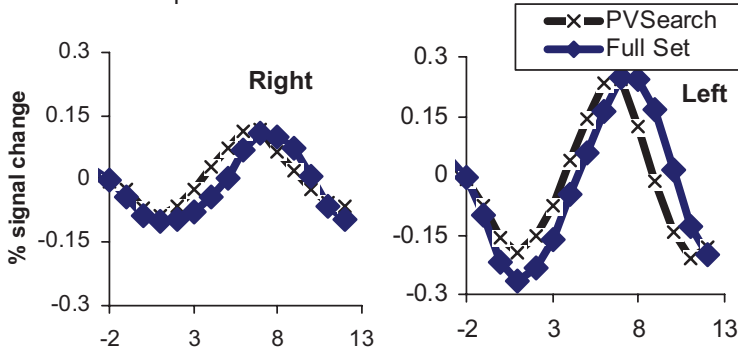

C: Half set - preview search
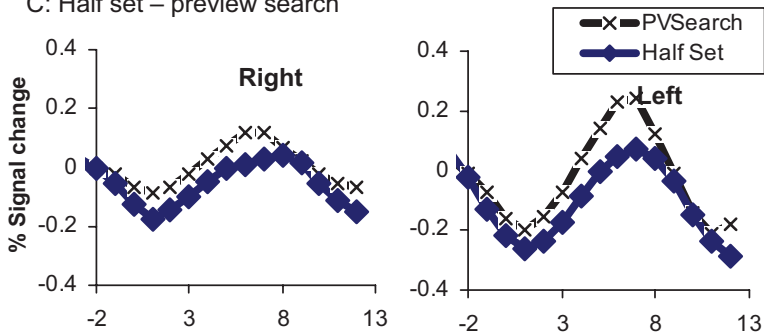

D: Preview - loaded preview search
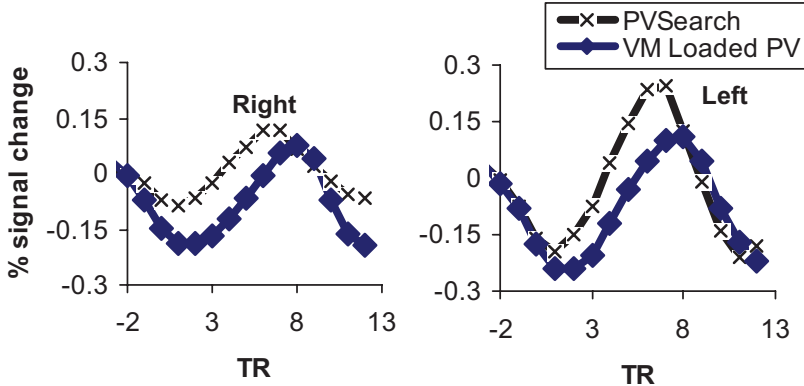

Figure 4. Activation plotted against time to repetition (TR) from the left and right fusiform face area averaged over all participants. Each subplot shows a different contrast and hemisphere. Crosses show activations from the conditions showing preview benefit, and diamonds show activations from conditions without preview. PVSearch = preview search; VM Loaded PV $=$ preview search done concurrently with a visual memory task (i.e., loaded preview). 


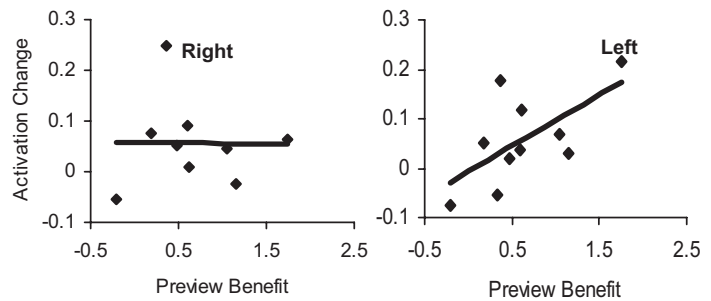

Figure 5. Magnitude of preview benefit plotted against average blood oxygen level dependent change in face-processing areas. Each point represents 1 participant's data.

related to adopting a narrow mode of attention but perhaps are more due to competition for resources with the preview.

The fMRI analysis showed a number of important results. First, there was increased activation in content-specific stimulus processing areas associated with successful use of the preview, and this occurred even when the search displays were omitted. In the critical comparison between the preview-only and dummy-only trials, the displays were identical and the only difference was in the attentional set of the participants, who knew that on the majority of trials in the preview condition, the preview remained constant and could be actively ignored. Thus, the increased activation linked to the preview is a result of the internal state of the participants and not of the stimulus.

Second, replicating prior fMRI experiments on visual search (Olivers et al., 2005; Pollmann et al., 2003), we found increased activation in the preview condition in a network of posterior brain areas. Brain regions in the precuneus, cuneus, and cerebellum were consistently activated in contrasts between the preview and the other search conditions, including contrasts made when only the preview was presented (in the preview-only vs. the dummy-only contrast)

Third, brain regions associated with our visual working memory task overlapped considerably with brain regions associated with the preview benefit (particularly the precuneus). The increased activity in the preview condition in the fusiform face area was linked to the specific content of the face previews. This activation decreased when a VM task interfered with the preview benefit, whereas the magnitude of the increase in activation (in the preview-only relative to the dummy-only condition) was correlated with the preview benefit on search performance. Because the preview is ignored and participants behave as if they are attending to just the new items, traditional models of attention predict that the activations in the stimulus-specific regions would be reduced, and, indeed, decreases in activation have previously been found when participants ignore stimuli (Gazzaley et al., 2005; A. T. Smith, Singh, \& Greenlee, 2000). However, the precise relationship of the BOLD signal to neural processes remains unclear, and increases in activation may come from both increases in cell firing and/or from the inhibition of some cells (which may consequently require more oxygen). It is thus difficult to tell from the imaging data alone whether the activation reflects an enhancement of the representation of the preview or inhibition. Behavioral data using probe dot detection, however, suggest that, at least initially, previews are attended and actively encoded (Humphreys et al., 2004), and a parsimonious account of these data is that activation of the preview's representation enables the items involved to be actively ignored. The evidence for enhanced activity linked to the contents of the preview, rather than the category of item expected in the search task, also supports the idea of active suppression rather than active anticipation of the search display (cf. Braithwaite \& Humphreys, 2003).

In addition to activation in content-specific regions of the cortex based on the contents of the preview, we also found activation in the posterior parietal cortex (cuneus and precuneus). This activation overlapped with cortical regions activated in the VM task, providing direct evidence for their role in representing the locations of items in the preview. Furthermore, this neural overlap can also provide an account of the interference effect from the VM task on preview search-essentially, if neural representations are given over to the VM task, then the neural representation of the preview will be less well-established and less easy to actively filter out from behavior. There is one concern with the behavioral data on the effects of working memory, however, which is that it generated an effect on the intercept of the search function (the loaded preview condition being overall slower than the standard preview condition; there was an increase in the slope of the function by about $10 \mathrm{~ms} /$ item, but this was not reliable). This could be interpreted in terms of the working memory task affecting a process other than search itself-for example, the initiation of search might be delayed because of the requirement to disengage attention from the memory task. The imaging data are useful here, though, because they show that there is less activation associated with the contents of the preview when participants are in the memory task condition. A task-switching account of this reduction in activation in the preview condition seems unlikely. Our conclusion is that the primary effect of the secondary task is to prevent the previewed items from being actively ignored, leaving the previewed items to compete for selection with items in the preview. This tended to increase the slopes as well as the intercepts of the search functions.

\section{Conclusions}

We have presented behavioral and fMRI evidence on the processes underlying preview search. Our behavioral data demonstrate that a preview benefit can be established with relatively complex photographic images and that there is selective interference on preview search from a secondary visual working memory task (even when the items for the task are presented prior to the preview appearing). Our imaging studies support earlier experiments indicating that preview search is associated with increased activation in the posterior parietal cortex, particularly in the left hemisphere (Olivers et al., 2005; Pollmann et al., 2003). In addition, we advanced these prior studies by showing that (a) there was enhanced activation in regions of the cortex responding to the content of the preview, specifically when participants were set to ignore the preview; (b) that this enhanced activation in contentspecific regions correlated with the behavioral benefit in preview search; and (c) that the visual working memory task activated areas of the posterior parietal cortex also associated with the preview condition. This last result provides converging evidence for the encoding of the spatial representations of preview items in preview search while also indicating that a reason for secondary task interference is that demands on visual working memory can compete with demands from the preview for spatial representation. 
These data add not only to our understanding of the neural basis of preview search but also to functional accounts of performance. In particular, we found evidence for enhanced activity of the properties of the preview in a condition in which only the preview appeared when these trials were embedded in the context of preview search. This enhanced activity predicted the preview benefit on search performance. Neither of these results is predicted by accounts that propose that the preview benefit is due solely to attentional capture by new onsets (Donk \& Theeuwes, 2001) or automatic temporal segmentation and attention to new items (Jiang et al., 2002). The data also provide no support for the idea that participants use the preview to predict the upcoming search items because then activity should have been linked to the search stimuli (e.g., houses) and not the previews (faces). Allen and Humphreys (2007) have also recently provided psychophysical evidence against the idea that the preview benefit is due to participants actively predicting the spatial locations of new items from the locations occupied by previewed stimuli. In addition to a standard preview condition (previewed items presented prior to the new search stimuli), they had a condition in which participants were cued to the upcoming locations of stimuli in the search task (equivalent to the new items in preview search). Uncued items then appeared at the locations that were occupied by previewed items in standard preview search. Search in this precue condition was worse than in the standard preview condition. That is, it was easier to eliminate previewed items from search than items in (uncued) locations. Active anticipation of the new stimuli alone is not sufficient to account for the preview benefit.

The imaging data also indicate that, in addition to any inhibition of the locations of previewed items, there is also processing linked to the content of these stimuli. Previous behavioral studies have provided evidence for features, such as the colors, of the previewed items being suppressed. Thus, new targets sharing the color of the previewed (to-be-ignored) items are difficult to detect under preview conditions - a negative feature-based carryover effect (Braithwaite et al., 2005; Olivers \& Humphreys, 2003). Olivers and Humphreys (2003) found that this result occurred even when the previewed items were switched off at the onset of the new stimuli, whereas Braithwaite et al. (2005) reported the effect even when the previewed items changed color when the new stimuli appeared. These results indicate the effect is indeed due to the carryover of inhibition from preview to search items and not to grouping of the new target with the old search items, for example. Here, we show effects linked to the category of the old stimuli (whether these items are faces or houses), or, at the very least, collections of features, not only to simple feature properties. We conclude that the prioritization of new items is enhanced by the suppression of all properties of previewed items that distinguish these stimuli from the new search targets. Whether this extends to even higher levels (e.g., the semantic properties of items) is a question open to future research.

Although we have argued that the neural enhancement of responses to the contents of the preview provide a neural marker of active ignoring, the present data do not provide information directly on the neural basis of suppressing old items. Prior behavioral data (Humphreys et al., 2004) suggest that there is initially enhanced attention to the preview when it is to be actively ignoredperhaps to refine spatial encoding and to derive the properties of the preview that are to be filtered during subsequent search. This is followed by a bias against the previewed stimuli. The increased activation, evident in the preview condition here, may reflect the stage of enhanced processing characteristic of processing soon after the onset of the preview. Alternatively, it may reflect the application of suppression to the preview and increased blood flow in response to this suppression. In either case, the data illustrate that there is differential active processing of the preview under conditions of preview search linked to the magnitude of the preview benefit

Of course, the ability to ignore stimuli has also been investigated using experimental paradigms other than preview search. For example, aspects of active ignoring may be found in studies of cuing attention, Stroop interference, and negative priming (to name but three). Cuing attention away from a stimulus can lead to reduced performance and a decrease in BOLD activation (Gazzaley et al., 2005; A. T. Smith et al., 2000). In a recent fMRI study of the Stroop task, Egner and Hirsch (2005) compared activation when participants had to attend to face stimuli or to ignore congruent or incongruent faces while reading a name. Activation in face-specific areas was increased when participants attended to the face images. When the faces were ignored, however, there was no change (neither an increase nor a decrease) in face-specific activation. This implies that these paradigms are not measuring the same processes as measured by the preview benefit.

A few studies have found increases of brain activation linked to ignoring stimuli. For example, Serences et al. (2004) found greater activation in visual areas when participants expected more distracters in a search display. In a study of negative priming, Wright et al. (2006) found increases in activation in visual areas when targets occupied locations that previously held a distracter. They did not find this pattern of activation in interleaved positive priming trials (i.e., targets in positions previously occupied by a target). Thus, activation increased when participants needed to ignore a location. This appears to be consistent with our finding of increasing activation associated with the preview benefit; however, the increase in activation with negative priming was associated with slower RTs and could reflect more difficult processing on negative priming trials. In contrast, here the increase in activation is associated with faster search performance and thus cannot be attributed to the greater difficulty of the particular condition. Ongoing work in our laboratory is concerned with comparing fMRI and behavior in different paradigms to find commonalities.

Finally, we note the contribution to a functional account of performance here from functional brain imaging. In the present study, the imaging data provide an extra constraint linked to localization of activation in the brain, which tells us about whether there is activity linked to the preview or the upcoming search display and whether interference on search occurs under conditions where common brain regions are activated in the primary and secondary tasks. This highlights the utility of functional imaging for understanding not only the localization of cognitive function but also the underlying functional processes themselves (see Humphreys \& Price, 2001).

\section{References}

Agter, F., \& Donk, M. (2005). Prioritized selection in visual search through onset capture and color inhibition: Evidence from a probe-dot detection task. Journal of Experimental Psychology: Human Perception and Performance, 31, 722-730. 
Allen, H. A., \& Humphreys, G. W. (2007). A psychophysical investigation into the preview benefit in visual search. Vision Research, 47, 735-745.

Beckman, C., Jenkinson, M., \& Smith, S. M. (2003). General multi-level linear modelling for group analysis in fMRI. Neuroimage, 20, 10521063.

Braithwaite, J. J., \& Humphreys, G. W. (2003). Inhibition and anticipation in visual search: Evidence from effects of color foreknowledge on preview search. Perception \& Psychophysics, 65, 213-237.

Braithwaite, J. J., Humphreys, G. W., \& Hulleman, J. (2005). Color-based grouping and inhibition in visual search: Evidence from a probe detection analysis of preview search. Perception \& Psychophysics, 67, 81101.

Chambers, C., Mark, G., Stokes, M. G., Janko, N. E., \& Mattingley, J. B. (2006). Enhancement of visual selection during transient disruption of parietal cortex. Brain Research, 1097, 149-155.

Donk, M., \& Theeuwes, J. (2001). Visual marking beside the mark: Prioritizing selection by abrupt onsets. Perception \& Psychophysics, 63, 891-900

Egner, T., \& Hirsch, J. (2005). Cognitive control mechanisms resolve conflict through cortical amplification of task relevant information. Nature Neuroscience, 8, 1784-1790.

Gazzaley, A., Cooney, J. W., McEvoy, K., Knight, R. T., \& D’Esposito, M. (2005). Top-down enhancement and suppression of the magnitude and speed of neural activity. Journal of Cognitive Neuroscience, 17, 507517.

Humphreys, G. W., Jung Stalmann, B., \& Olivers, C. N. L. (2004). An analysis of the time course of attention in preview search. Perception \& Psychophysics, 66, 713-730.

Humphreys, G. W., \& Price, C. J. (2001). Cognitive neuropsychology and functional brain imaging: Implications for functional and anatomical models of cognition. Acta Psychologica, 107, 119-153.

Humphreys, G. W., Watson, D. G., \& Jolicoeur, P. (2002). Fractionating the preview benefit in search: Dual-task decomposition of visual marking by timing and modality. Journal of Experimental Psychology: Human Perception and Performance, 28, 640-660.

Jenkinson, M., Bannister, J. M., \& Smith, A. (2005). Improved optimisation for the robust and accurate linear registration and motion correction of brain images. Neuroimage, 17, 825-841.

Jenkinson, M., \& Smith, S. M. (2001). A global optimisation method for robust affine registration of brain images. Medical Image Analysis, 5, $143-156$.

Jiang, Y. H., Chun, M. M., \& Marks, L. E. (2002). Visual marking: Selective attention to asynchronous temporal groups. Journal of Experimental Psychology: Human Perception and Performance, 28, 717-730.

Kanwisher, N., McDermott, J., \& Chun, M. M. (1997). The fusiform face area: A module in human extra striate cortex specialized for face perception. Journal of Neuroscience, 17, 4302-4311.

Lavie, N. (1995). Perceptual load as a necessary condition for selective attention. Journal of Experimental Psychology: Human perception and performance, 21, 451-468.

Mevorach, C., Humphreys, G. W., \& Shalev, L. (2006). Opposite biases in salience-based selection for the left and right posterior parietal cortex. Nature Neuroscience, 9, 740-742.

Neisser, U. (1976). Cognition and reality: Principals and implications of cognitive psychology. San Francisco: W. H. Freeman and Company.

O'Craven, K. M., Downing, P. E., \& Kanwisher, N. (1999, October 7). fMRI evidence for objects as the units of attentional selection. Nature, 401, 584-587.

Olivers, C. N. L., \& Humphreys, G. W. (2002). When visual marking meets the attentional blink: More evidence for top-down, limitedcapacity inhibition. Journal of Experimental Psychology: Human Perception and Performance, 28, 22-42.

Olivers, C. N. L., \& Humphreys, G. W. (2003). Visual marking inhibits singleton capture. Cognitive Psychology, 47, 1-42.

Olivers, C. N. L., Humphreys, G. W., \& Braithwaite, J. J. (2006). The preview search task: Evidence for visual marking. Visual Cognition, 14, $716-735$.

Olivers, C. N. L., Smith, S., Matthews, P., \& Humphreys, G. W. (2005). Prioritizing new over old: An fMRI study of the preview search task. Human Brain Mapping, 24, 69-78.

Pessoa, L., Gutierrez, E., Bandettini, P. A., \& Ungerleider, L. G. (2002). Neural correlates of visual working memory: fMRl amplitude predicts task performance. Neuron, 35, 975-987.

Pollmann, S., Weidner, R., Humphreys, G. W., Olivers, C. N. L., Muller, K., Lohmann, G., et al. (2003). Separating distractor rejection and target detection in posterior parietal cortex: An event-related fMRI study of visual marking. Neuroimage, 18, 310-323.

Schmahmann, J. D., Doyon, J., Toga, A. W., Petrides, M., \& Evans, A. C. (2000). MRI atlas of the human cerebellum. London: Academic Press.

Serences, J. T., Yantis, S., Culberson, A., \& Awh, E. (2004). Preparatory activity in visual cortex indexes distractor suppression during covert spatial orienting. Journal of Neurophysiology, 92, 3538-3545.

Smith, A. T., Singh, K. D., \& Greenlee, M. W. (2000). Attentional suppression of activity in the human visual cortex. NeuroReport, 11, 271-277.

Smith, S. (2002). Fast robust automated brain extraction. Human Brain Mapping, 17, 143-155.

Townsend, J. T., \& Ashby, F. G. (1983). Stochastic modelling of elementary psychological processes. London: Cambridge University Press.

Treisman, A. M., \& Gelade, G. (1980). A feature-integration theory of attention. Cognitive Psychology, 12, 97-136.

Watson, D. G., \& Humphreys, G. W. (1997). Visual marking: Prioritizing selection for new objects by top-down attentional inhibition of old objects. Psychological Review, 104, 90-122.

Watson, D. G., \& Humphreys, G. W. (2000). Visual marking: Evidence for inhibition using a probe-dot detection paradigm. Perception \& Psychophysics, 62, 471-481.

Woolrich, M. W., Behrens, T. E. J., Bedell, H. E., Jenkinson, M., \& Smith, S. M. (2004). Multi-level linear modelling for fMRI group analysis using Bayesian inference. Neuroimage, 21, 1732-1747.

Woolrich, M. W., Ripley, B. D., Brady, J. M., \& Smith, S. M. (2001) Temporal autocorrelation in univariate linear modelling of fMRI data. Neuroimage, 14, 1370-1386.

Worsley, K. J., Evans, A. C., Marrett, S., \& Nelson, C. A. (1992). A three-dimensional statistical analysis for CBF activation studies in human brain. Journal of Cerebral Blood Flow and Metabolism, 12, 900918.

Wright, C. I., Keuthen, N. J., Savage, C. R., Martis, B., Williams, D., Wedig, M., et al. (2006). Brain correlates of negative and positive visuospatial priming in adults. Neuroimage 30, 983-991.

Yantis, S., \& Jonides, J. (1990). Abrupt visual onsets and selective attention: Voluntary versus automatic allocation. Journal of Experimental Psychology: Human Perception and Performance, 16, 121-134.

Received January 31, 2007

Revision received July 24, 2007

Accepted August 6, 2007 